\title{
Grounded theory analysis
}

David F Marks

Potential competing interests: The author(s) declared that no potential competing interests exist.

Grounded theory analysis is a set of guidelines for conducting qualitative data analysis. 